\title{
Autoimmune/Inflammatory Syndrome Induced by Adjuvants and Thyroid Autoimmunity
}

\author{
Abdulla Watad ${ }^{1,2,3}$, Paula David ${ }^{2,4}$, Stav Brown ${ }^{3}$ and Yehuda Shoenfeld ${ }^{2,3,5 *}$ \\ 'Department of Medicine "B", Sheba Medical Center, Tel-Aviv, Israel, '2Zabludowicz Center for Autoimmune Diseases, Sheba \\ Medical Center, Tel-Aviv, Israel, ${ }^{3}$ Sackler Faculty of Medicine, Tel-Aviv University, Tel-Aviv, Israel, ${ }^{4}$ Faculdade de Ciências \\ Médicas, Universidade do Estado do Rio de Janeiro, Rio de Janeiro, Brazil, ${ }^{5}$ Laura Schwarz-Kipp Chair for Research of \\ Autoimmune Diseases, Tel-Aviv University, Tel-Aviv, Israel
}

\section{OPEN ACCESS}

Edited by: Alessandro Antonelli, University of Pisa, Italy

Reviewed by:

Silvia Martina Ferrari, University of Pisa, Italy Illaria Ruffili, University of Pisa, Italy Carlo Perricone, Sapienza University, Italy

*Correspondence: Yehuda Shoenfeld shoenfe/@post.tau.ac.il

Specialty section: This article was submitted to Thyroid Endocrinology, a section of the journal Frontiers in Endocrinology

Received: 12 October 2016 Accepted: 21 November 2016 Published: 24 January 2017

Citation:

Watad A, David P, Brown S and Shoenfeld Y (2017) Autoimmune/ Inflammatory Syndrome Induced by Adjuvants and Thyroid Autoimmunity.

Front. Endocrinol. 7:150. doi: 10.3389/fendo.2016.00150
The autoimmune/inflammatory syndrome induced by adjuvants (ASIA), presented by Shoenfeld and Agmon-Levin in 2011, is an entity that incorporates diverse autoimmune conditions induced by the exposure to various adjuvants. Adjuvants are agents that entail the capability to induce immune reactions. Adjuvants are found in many vaccines and used mainly to increase the response to vaccination in the general population. Silicone has also been reported to be able to induce diverse immune reactions. Clinical cases and series of heterogeneous autoimmune conditions including systemic sclerosis, systemic lupus erythematosus, and rheumatoid arthritis have been reported to be induced by several adjuvants. However, only a small number of cases of autoimmune thyroid disorder have been included under the umbrella of ASIA syndrome. Indeed, clinical cases of Hashimoto's thyroiditis and/or subacute thyroiditis were observed after the exposure to vaccines as well as silicone implantation. In our review, we aimed to summarize the current knowledge on ASIA syndrome presented as endocrinopathies, focusing on autoimmune thyroid disorders associated with the various adjuvants.

Keywords: autoimmune/inflammatory syndrome induced by adjuvants, thyroid, endocrinopathy, adjuvants, vaccines, silicone, Hashimoto's thyroiditis, Graves disease

\section{INTRODUCTION}

Adjuvants are substances that are able to trigger autoimmunity via a variety of mechanisms, such as alteration of the host's immune system, polyclonal activation of B cells, effects on cellular immunity, immunoregulatory cells, viral-induced antibodies, and acceleration of molecular mimicry (1). Exposure to adjuvants can occur in a variety of methods due to their wide range of uses in vaccines, mineral oils, silicone implants, and many other products and devices. The association between adjuvant exposure and autoimmunity manifests itself in five autoimmune conditions sharing similar autoimmunity manifestations $(2,3)$, such as the postvaccination phenomena, the macrophagic myofasciitis syndrome (MMF), the Gulf war syndrome (GWS), siliconosis, and the sick building syndrome (SBS) $(4,5)$. The autoimmune/inflammatory syndrome induced by adjuvants (ASIA), presented by Shoenfeld and Agmon-Levin (6) in 2011, is a single entity that incorporates all five conditions. Extensive research has identified the genetic background, contributing to the development of ASIA syndrome in predisposed individuals following adjuvant exposure. A large number of autoimmune diseases share several alleles of the HLA class II such as DRB1 locus. The development 
of specific autoantibodies is determined by DRB1 alleles leading to an abnormal response and development of full-blown autoimmune diseases $(7,8)$.

When used in vaccines, adjuvants are purposely used as immunogenicity enhancing agents that are essential for directing the adaptive immunoresponse (9). However, they might also trigger undesired autoimmune reactions that question the use of adjuvants and their safety in the context of $\mathrm{DRB}^{\star} 01$ genetic background (10).

A systematic review by Jara et al. (4) reported that 4479 ASIA cases have been identified since its presentation in 2011. Among them, 305 were considered severe, with the majority of these cases being developed following vaccines mainly directed to HPV, HBV, and seasonal influenza. Despite vaccines' proven record of safety and efficiency, aluminum hydroxide was used in these vaccines along with the viral antigens as an adjuvant. Due to aluminum's capability to enhance the immunoresponse, it enables the usage of smaller amount of antigens. However, enhanced immunogenicity might lead to enhanced reactogenicity in a process not always benign involving pathological stimulation (11).

The other adjuvants containing products yielding severe clinical manifestations are silicone implants and mineral oil fillers $(4,12)$.

Silicone has been considered as an inert material, which is unable to induce immune reactions in the human body. Therefore, it has been used in many medical devices for the last 60 years, including both silicone and saline breast implants. However, a possible association between silicone exposure and autoimmune diseases has been reported in many studies demonstrating the development of autoimmune diseases and autoantibodies in patients following exposure to silicone implants $(13,14)$. Improved clinical manifestations after the extraction of implants (15) support the relationship between silicone and autoimmunity.

Mineral oil injections, which are prevalent in Mexico and Latin America for cosmetic uses, have been identified as a leading cause of ASIA syndrome as well via the proposed mechanism of chronic inflammation induction leading to granuloma formation and thickening of the dermis $(4,10)$.

The risk for autoimmune diseases, determined by the patient's genetic background, is increased in patients with autoimmune diseases history such as type 1 diabetes mellitus (T1DM). Thyroid antibodies can be identified in approximately $20-25 \%$ of patients with type 1 diabetes, and up to $50 \%$ of them progress to clinical autoimmune thyroid disease (AITD) (16). Thyroid autoimmune diseases have been described in many case reports and case series, presenting thyroid autoimmune manifestations along with other autoimmune conditions.

In genetically predisposed individuals, under particular conditions, molecular mimicry between microbial and human antigens has been shown to be able to turn a defensive immunoresponse into autoimmune response. This mechanism has yet to be explored in the field of thyroid autoimmune diseases (17). In our review, we aimed to summarize the current knowledge about ASIA syndrome and the relationship between adjuvants and autoimmune diseases, focusing on its association with autoimmune endocrinopathies and thyroid autoimmunity.

\section{ENDOCRINOPATHY AND ASIA SYNDROME}

Pathological processes of the endocrine glands result in abnormal levels of circulating hormones, which lead to endocrinopathies. Some endocrine disorders are immune mediated, such as Hashimoto's thyroiditis (HT), Graves' disease, and T1DM (18-20). Thus, it is possible that endocrine autoimmune diseases can be triggered by adjuvants, configuring cases of ASIA syndrome. Case reports, cohort and case-control studies on ASIA syndrome, and the majority of the endocrinopathies are still scarce. Lately, primary ovarian failure (POF) has been linked to ASIA, especially after vaccination (21-25).

Primary ovarian failure or premature ovarian insufficiency is defined as a combination of amenorrhea, for a minimum of 4 months, decline in sex steroids, and follicle-stimulating hormone (FSH) above $40 \mathrm{IU} / \mathrm{l}$ at two measurements with an interval of at least 1 month in women younger than 40 years (26). POF is a disorder with multiple etiologic mechanisms. The presence of lymphocytic invasion in the oophorus and the identification of autoantibodies against ovarium antigens on the theca, granulose, corpus luteum, and zona pellucida (27-29) support the idea that part of its etiology, estimated in $20-30 \%$ (30), is immune mediated. Furthermore, POF is commonly associated with other autoimmune diseases, including Addison's disease, thyroiditis, autoimmune polyglandular syndrome, systemic lupus erythematosus (SLE), hemolytic anemia, idiopathic thrombocytopenic purpura (ITP), and Sjogren's syndrome (31). The pathogenesis of POF also involves genetic mutations, metabolic disorders, and environmental factors, such as virus infection, chemo and radiotherapy, and surgeries (30).

HPV vaccine has been reported as an important issue in ASIA syndrome, already being related, for instance, to Guillain-Barré syndrome and other neuropathies, such as SLE, vasculitis, ITP, and autoimmune hepatitis (32-36). Developing autoimmune diseases as an adverse effect of the vaccine can be both due to its HPV virus-like particles, which have potent immuno-stimulatory properties (and can induce autoimmunity by molecular mimicry, epitope spreading, bystander activation, and polyclonal activation) (37), and due to the presence of aluminum as an adjuvant in the vaccine (38). Adjuvants are capable of increasing, intensifying, and prolonging antigen-specific immunoresponse of the vaccines without holding its own specific antigenic effect (38). Autoimmune well-defined diseases, as well as the nonspecific immune disorders, following vaccination can present as a subacute vaccination side effect or appear months or years after the boosters (39-43). Genetically predisposed patients are more likely to exhibit late manifestations and are in a higher risk of developing ASIA syndrome $(36,44)$.

Colafrancesco et al. (21) recently reported three cases of POF following immunization with HPV vaccine. The three patients fulfilled the criteria for ASIA syndrome suggested by Shoenfeld and Agmon-Levin (6). They described three young women, previously healthy and with normal sexual development, who received three administrations of the quadrivalent $\mathrm{HPV}$ vaccine. The patients experienced general symptoms, including nausea, stomachaches, heavy and burning sensations in the injected arm, 
headaches, insomnia, arthralgia, depression, anxiety, and difficulty in concentrating, and then presented amenorrhea within approximately 10 months, 2 years, and 10 years after the first dose. Two of them were positive for previously negative antibodies (anti-TPO and antiovarian). Hormonal screening was performed, showing increased FSH and luteinizing hormone (LH) plus extremely low levels of estradiol. Pregnancy was excluded, as well as no abnormalities were revealed in the transvaginal and pelvic ultrasound. After a karyotype evaluation and search for Fragile $\mathrm{X}$ syndrome with no aberrations, they were diagnosed with POF. Moreover, two of the three patients were siblings leading to the hypothesis that may exist as a rare risk factor for this adverse effect.

Little and Ward (22) also reported a case of POF succeeding HPV vaccination, in a 16-year-old patient, who presented irregular menses after taking the quadrivalent vaccine, followed by oligomenorrhea and amenorrhea. Her hormone profile also showed high levels of FSH and LH and low levels of estradiol and anti-mullerian hormone (AMH), and after excluding pregnancy and genetic, endocrinal, and other causes, she was diagnosed with POF.

Problems of quadrivalent HPV vaccine introduction in the market were wisely pointed by Little and Ward (25). They reported three other cases of young women who develop POF after having quadrivalent HPV vaccine and questioned some issues about its safety. First, despite the fact that the vaccine protocol suggests three doses, in the preclinical studies for toxicity, only two boosters were given to the rats. Still, the animals' reproductive system was not analyzed in a long-term period. Moreover, the phase II and III clinical studies on safety of the vaccine regarding the fertility were not complete: half of the subjects studied were lost to follow-up at 1 year; some of the subjects were on hormone contraception methods, which could mask the ovarian insufficiency; they have not considered medical conditions that flourished more than 7 months after the vaccination as associated with the vaccine; and adverse effects were only reported 2 weeks after the boosters. Furthermore, the placebo used as control in the phase III safety studies of the quadrivalent HPV vaccine was aluminum, also present in the vaccine solution, which was already shown to play as an adjuvant in ASIA syndrome.

Thus, HPV vaccine is likely to be an important trigger in ASIA syndrome, including immuno-mediated endocrine disorders, such as POF. Due to long periods of intervals between the vaccine injections and the development of the ovarian insufficiency, it is questionable if there is indeed a causal relationship between them. However, as previously mentioned, the safety preclinical and clinical studies of HPV vaccine are lacking some information regarding fertility safety, and the side effects were shown to be able to appear even after months or years.

Other vaccines and adjuvants may also trigger POF, as well as other immuno-mediated endocrinopathies, like for instance, type 1-diabetes may be induced by the same adjuvants. Indeed, in a cohort study with 211 young female patients with autoimmune diseases and 857 matched controls, they showed that patients exposed to quadrivalent HPV vaccine were in a higher risk of developing type 1 -diabetes mellitus $(\mathrm{OR}=1.2)(45)$. Additionally, it was shown in a prospective cohort study (46) that some vaccines are related to increased levels of diabetes autoantibodies, such as antibody against glutamic acid decarboxylase (GADA) and tyrosine phosphatase (IA-2A). These autoantibodies, which are considered reliable markers for the disease process $(47,48)$, were more frequently found in the subjects who received hemophilus influenza $B(\mathrm{HIB})$ vaccination $(\mathrm{OR}=5.9$ and 3.4 in IA-2A and GADA, respectively). Especially, the IA-2A serum concentrations were significantly higher in patients exposed to HIB. Also, BCG was correlated to an enhanced prevalence of IA-2A $(p<0.01)$. The previously mentioned studies suggest that ASIA syndrome, particularly post vaccination, and endocrinopathies might be linked.

\section{AUTOIMMUNE THYROID DISEASE AND ASIA SYNDROME}

During the last years, abundant case reports and series were published supporting that various autoimmune disorders may be induced by adjuvants and be enclosed under ASIA syndrome $(4,12)$. Despite the fact of being the most common autoimmune disorder, unexpectedly, we have revealed very few articles and case reports in the literature describing the induction of AITD by various adjuvants. In this section, we report that the relevant case descriptions of AITD were reported to be correlated to immunization and silicone implants.

Hernàn Martinez et al. (49) described a case of a 55-year-old man with a family history of autoimmune diseases and medical history of diabetes and psoriasis, who developed subacute thyroiditis shortly after the administration of an influenza vaccine. Subacute thyroiditis is a very rare disease, and the authors of the mentioned case concluded that the induction of the disease was a result of an interaction between the genetic predisposition and vaccination. Another similar case of subacute thyroiditis was reported in a 25 -year-old female (50). The patient was admitted due to fever, swelling, and tender mass in the neck. Two days before her presentation, she received influenza vaccine (Vaxigrip). Biopsy of the thyroid has revealed multinuclear giant cell granulomas.

A previously healthy 36-year-old female presented with clinical symptoms of thyrotoxicosis including tachycardia, anxiety, and tenderness in her neck (51). One month before her presentation, she received $\mathrm{H} 1 \mathrm{~N} 1$ vaccine. Thyroid function tests confirmed remarkable thyrotoxicosis. Thyroid scintigraphy was performed and showed significant diffuse reduction in the technetium uptake. Therefore, a diagnosis of subacute thyroiditis was made. Moving to another type of adjuvant, cases of granulomatous inflammation of the thyroid have been reported with silicone breast implants (52). Vayssairat et al. (53) described two cases of HT after receiving a silicone gel-filled breast implants. Both cases were induced after a long period of incubation, the first case is a 45-year-old women who had bilateral silicone implant of the breast in 1976 and developed HT in 1991. In addition, the patient complained of other non-specific symptoms including fatigue, morning stiffness, and sicca syndrome. Thyroid ultrasonography 
showed an enlarged thyroid gland with a diffusely hypoechogenic pattern. The implants were painful and removed, showing extremely dense connective tissue with fibrosis. The second case of HT presented with hyperthyroidism clinical manifestation, 10 years after the silicon implantation, reporting positive antiTPO. The implants were again painful, and the patient developed positive antinuclear antibodies (ANA). An animal experiment aimed to evaluate the immunological adjuvancy potential of silicone gel taken from breast implants (54). The study has found that silicone gel is able to stimulate the production of autoantibodies to rat thyroglobulin and bovine collagen II. However, this immune reaction was not associated with any histological evidence of thyroiditis or arthritis.

A cohort study was performed to assess the risk of new onset autoimmune disease in young women exposed to human papillomavirus-16/18 AS04-adjuvanted vaccine in the United Kingdom (55). The study reported an incidence rate ratio (95\% CI) of 3.75 (1.25-11.31) for autoimmune thyroiditis among females.

An animal study has reported that immunization of BALB/c mice with the extracellular domain of the human TSH receptor led to the production of TSH binding-inhibiting and thyroidblocking antibodies accompanied by lymphocytic infiltration of the thyroid (56).

\section{REFERENCES}

1. Perricone C, Colafrancesco S, Mazor RD, Soriano A, Agmon-Levin N, Shoenfeld Y. Autoimmune/inflammatory syndrome induced by adjuvants (ASIA) 2013: unveiling the pathogenic, clinical and diagnostic aspects. J Autoimmun (2013) 47:1-16. doi:10.1016/j.jaut.2013.10.004

2. Vera-Lastra O, Medina G, Cruz-Dominguez MDP, Ramirez P, Gayosso-Rivera JA, Anduaga-Dominguez $\mathrm{H}$, et al. Human adjuvant disease induced by foreign substances: a new model of ASIA (Shoenfeld's syndrome). Lupus (2012) 21:128-35. doi:10.1177/0961203311429317

3. Meroni PL. Autoimmune or auto-inflammatory syndrome induced by adjuvants (ASIA): old truths and a new syndrome? J Autoimmun (2011) 36:1-3. doi:10.1016/j.jaut.2010.10.004

4. Jara LJ, García-Collinot G, Medina G, Cruz-Dominguez MD, Vera-Lastra O, Carranza-Muleiro RA, et al. Severe manifestations of autoimmune syndrome induced by adjuvants (Shoenfeld's syndrome). Immunol Res (2016). doi:10.1007/s12026-016-8811-0

5. Israeli E, Pardo A. The sick building syndrome as a part of the autoimmune (auto-inflammatory) syndrome induced by adjuvants. Mod Rheumatol (2011) 21:235-9. doi:10.1007/s10165-010-0380-9

6. Shoenfeld Y, Agmon-Levin N. "ASIA" - autoimmune/inflammatory syndrome induced by adjuvants. JAutoimmun (2011) 36:4-8. doi:10.1016/ j.jaut.2010.07.003

7. Israeli E, Agmon-Levin N, Blank M, Shoenfeld Y. Macrophagic myofaciitis a vaccine (alum) autoimmune-related disease. Clin Rev Allergy Immunol (2011) 41:163-8. doi:10.1007/s12016-010-8212-4

8. Arango M-T, Perricone C, Kivity S, Cipriano E, Ceccarelli F, Valesini G, et al. HLA-DRB1 the notorious gene in the mosaic of autoimmunity. Immunol Res (2016):1-17. doi:10.1007/s12026-016-8817-7

9. Agmon-Levin N, Hughes GR, Shoenfeld Y. The spectrum of ASIA: "autoimmune (auto-inflammatory) Syndrome induced by adjuvants”. Lupus (2012) 21:118-20. doi:10.1177/0961203311429316

10. Vera-Lastra O, Medina G, Cruz-Dominguez MDP, Jara LJ, Shoenfeld Y. Autoimmune/inflammatory syndrome induced by adjuvants (Shoenfeld's syndrome): clinical and immunological spectrum. Expert Rev Clin Immunol (2013) 9:361-73. doi:10.1586/eci.13.2
In summary, ASIA syndrome is being more recognized by physicians, and therefore, more studies and cases have reported the correlation of the exposure to various adjuvants with diverse autoimmune diseases. Still, very few clinical reports and animal models studies were published to show the relationship between endocrinopathies in general and AITD in particular with adjuvants. However, the clinical cases of HT and/or subacute thyroiditis were observed after the exposure to vaccines as well as silicone implantation. Therefore, we believe that the minority of cases is not owing to rarity of association between adjuvants and AITD rather than the lack of awareness among physicians of such association. Consequently, physicians must be mindful that thyroiditis and other thyroid disorders can be induced by diverse adjuvants and therefore to reconsider non-essential vaccination in genetically predisposed individuals for autoimmune diseases.

\section{AUTHOR CONTRIBUTIONS}

$\mathrm{AW}, \mathrm{PD}, \mathrm{SB}$, and YS designed the study and reviewed the literature on ASIA syndrome and thyroid autoimmunity. AW, PD, and SB wrote the manuscript. AW and YS edited the manuscript. All the authors have revised the paper and approved the final edition.

11. Inbar R, Weiss R, Tomljenovic L, Arango MT, Deri Y, Shaw CA, et al. Behavioral abnormalities in young female mice following administration of aluminum adjuvants and the human papillomavirus (HPV) vaccine Gardasil. Vaccine (2016) pii:S0264-410X(16)00016-5. doi:10.1016/j.vaccine.2015.12.067

12. Colaris MJL, de Boer M, van der Hulst RR, Cohen Tervaert JW. Two hundreds cases of ASIA syndrome following silicone implants: a comparative study of 30 years and a review of current literature. Immunol Res (2016). doi:10.1007/ s12026-016-8821-y

13. Muzaffar AR, Rohrich RJ. The silicone gel-filled breast implant controversy: an update. Plast Reconstr Surg (2002) 109:742-7; quiz 748. doi:10.1097/00006534-200202000-00049

14. Bar-Meir E, Teuber SS, Lin HC, Alosacie I, Goddard G, Terybery J, et al. Multiple autoantibodies in patients with silicone breast implants. J Autoimmun (1995) 8:267-77. doi:10.1006/jaut.1995.0020

15. Maijers MC, de Blok CJM, Niessen FB, van der Veldt AAM, Ritt MJPF, Winters HAH, et al. Women with silicone breast implants and unexplained systemic symptoms: a descriptive cohort study. Neth J Med (2013) 71:534-40.

16. Kahaly GJ, Hansen MP. Type 1 diabetes associated autoimmunity. Autoimmun Rev (2016) 15:644-8. doi:10.1016/j.autrev.2016.02.017

17. Oldstone MBA. Molecular mimicry and autoimmune disease. Cell (1987) 50:819-20. doi:10.1016/0092-8674(87)90507-1

18. Prabhakar BS, Bahn RS, Smith TJ. Current perspective on the pathogenesis of Graves' disease and ophthalmopathy. Endocr Rev (2003) 24:802-35. doi:10.1210/er.2002-0020

19. Hollowell JG, Staehling NW, Flanders WD, Hannon WH, Gunter EW, Spencer CA, et al. Serum TSH, T(4), and thyroid antibodies in the United States population (1988 to 1994): National Health and Nutrition Examination Survey (NHANES III). J Clin Endocrinol Metab (2002) 87:489-99. doi:10.1210/ jcem.87.2.8182

20. Atkinson MA, Maclaren NK. The pathogenesis of insulin-dependent diabetes mellitus. $N$ Engl JMed (1994) 331:1428-36. doi:10.1056/ NEJM199411243312107

21. Colafrancesco S, Perricone C, Tomljenovic L, Shoenfeld Y. Human papilloma virus vaccine and primary ovarian failure: another facet of the autoimmune/ inflammatory syndrome induced by adjuvants. Am J Reprod Immunol (2013) 70:309-16. doi:10.1111/aji.12151 
22. Little DT, Ward HRG. Premature ovarian failure 3 years after menarche in a 16-year-old girl following human papillomavirus vaccination. BMJ Case Rep (2012) 2012:2-4. doi:10.1136/bcr-2012-006879

23. Gruber N, Shoenfeld Y. A link between human papilloma virus vaccination and primary ovarian insufficiency: current analysis. Curr Opin Obstet Gynecol (2015) 27:265-70. doi:10.1097/GCO.0000000000000183

24. Hawkes D, Buttery JP. Human papillomavirus vaccination and primary ovarian insufficiency: an association based on ideology rather than evidence. Curr Opin Obstet Gynecol (2016) 28:70-2. doi:10.1097/GCO.0000000000 000240

25. Little DT, Ward HRG. Adolescent premature ovarian insufficiency following human papillomavirus vaccination: a case series seen in general practice. J Investig Med High Impact Case Rep (2014) 2:2324709614556129. doi:10.1177/2324709614556129

26. Nelson LM. Clinical practice. Primary ovarian insufficiency. $N$ Engl J Med (2009) 360:606-14. doi:10.1056/NEJMcp0808697

27. Kelkar RL, Meherji PK, Kadam SS, Gupta SK, Nandedkar TD. Circulating auto-antibodies against the zona pellucida and thyroid microsomal antigen in women with premature ovarian failure. J Reprod Immunol (2005) 66:53-67. doi:10.1016/j.jri.2005.02.003

28. Chattopadhyay D, Sen MR, Katiyar P, Pandey LK. Antiovarian antibody in premature ovarian failure. Indian J Med Sci (1999) 53:254-8.

29. Mande PV, Parikh FR, Hinduja I, Zaveri K, Vaidya R, Gajbhiye R, et al. Identification and validation of candidate biomarkers involved in human ovarian autoimmunity. Reprod Biomed Online (2011) 23:471-83. doi:10.1016/ j.rbmo.2011.06.013

30. Petrikova J, Lazurova I. Ovarian failure and polycystic ovary syndrome. Autoimmun Rev (2012) 11:A471-8. doi:10.1016/j.autrev.2011.11.010

31. Hoek A, Schoemaker J, Drexhage HA. Premature ovarian failure and ovarian autoimmunity. Endocr Rev (1997) 18:107-34. doi:10.1210/er.18.1.107

32. Pugnet G, Ysebaert L, Bagheri H, Montastruc J-L, Laurent G. Immune thrombocytopenic purpura following human papillomavirus vaccination. Vaccine (2009) 27:3690. doi:10.1016/j.vaccine.2009.04.004

33. Souayah N, Michas-Martin PA, Nasar A, Krivitskaya N, Yacoub HA, Khan H, et al. Guillain-Barré syndrome after Gardasil vaccination: data from Vaccine Adverse Event Reporting System 2006-2009. Vaccine (2011) 29:886-9. doi:10.1016/j.vaccine.2010.09.020

34. Alvarez-Soria MJ, Hernandez-Gonzalez A, Carrasco-Garcia de Leon S, del Real-Francia MA, Gallardo-Alcaniz MJ, Lopez-Gomez JL. [Demyelinating disease and vaccination of the human papillomavirus]. Rev Neurol (2011) 52:472-6.

35. Gomes SM, Glover M, Malone M, Brogan P. Vasculitis following HPV immunization. Rheumatology (Oxford) (2013) 52:581-2. doi:10.1093/ rheumatology/kes168

36. Gatto M, Agmon-Levin N, Soriano A, Manna R, Maoz-Segal R, Kivity S, et al. Human papillomavirus vaccine and systemic lupus erythematosus. Clin Rheumatol (2013) 32:1301-7. doi:10.1007/s10067-013-2266-7

37. Sutton I, Lahoria R, Tan I, Clouston P, Barnett M. CNS demyelination and quadrivalent HPV vaccination. Mult Scler (2009) 15:116-9. doi: $10.1177 / 1352458508096868$

38. Israeli E, Agmon-Levin N, Blank M, Shoenfeld Y. Adjuvants and autoimmunity. Lupus (2009) 18:1217-25. doi:10.1177/0961203309345724

39. Couette M, Boisse MF, Maison P, Brugieres P, Cesaro P, Chevalier X, et al. Long-term persistence of vaccine-derived aluminum hydroxide is associated with chronic cognitive dysfunction. J Inorg Biochem (2009) 103:1571-8. doi:10.1016/j.jinorgbio.2009.08.005

40. Mikaeloff Y, Caridade G, Suissa S, Tardieu M. Hepatitis B vaccine and the risk of CNS inflammatory demyelination in childhood. Neurology (2009) 72:873-80. doi:10.1212/01.wnl.0000335762.42177.07

41. Shivane A, Hilton DA, Moate RM, Bond PR, Endean A. Macrophagic myofasciitis: a report of second case from UK. Neuropathol Appl Neurobiol (2012) 38:734-6. doi:10.1111/j.1365-2990.2012.01293.x
42. Poser CM, Behan PO. Late onset of Guillain-Barré syndrome.J Neuroimmunol (1982) 3:27-41. doi:10.1016/0165-5728(82)90016-9

43. Gherardi RK, Authier F-J. Macrophagic myofasciitis: characterization and pathophysiology. Lupus (2012) 21:184-9. doi:10.1177/0961203311429557

44. Zafrir Y, Agmon-Levin N, Paz Z, Shilton T, Shoenfeld Y. Autoimmunity following hepatitis B vaccine as part of the spectrum of "autoimmune (auto-inflammatory) syndrome induced by adjuvants" (ASIA): analysis of 93 cases. Lupus (2012) 21:146-52. doi:10.1177/0961203311429318

45. Grimaldi-Bensouda L, Guillemot D, Godeau B, Bénichou J, Lebrun-Frenay C, Papeix C, et al. Autoimmune disorders and quadrivalent human papillomavirus vaccination of young female subjects. J Intern Med (2014) 275:398-408. doi:10.1111/joim. 12155

46. Wahlberg J, Fredriksson J, Vaarala O, Ludvigsson J, Abis Study Group. Vaccinations may induce diabetes-related autoantibodies in one-year-old children. Ann N Y Acad Sci (2003) 1005:404-8. doi:10.1196/annals.1288.068

47. Bingley PJ, Bonifacio E, Williams AJK, Genovese S, Bottazzo GF, Gale EA. Prediction of IDDM in the general population: strategies based on combinations of autoantibody markers. Diabetes (1997) 46:1701-10. doi:10.2337/ diab.46.11.1701

48. Samuelsson UU, Sundkvist GG, Borg HH, Fernlund PP, Ludvigsson JJ. Islet autoantibodies in the prediction of diabetes in school children. Diabetes Res Clin Pract (2001) 51:51-7. doi:10.1016/S0168-8227(00)00199-6

49. Hernán Martinez J, Corder E, Uzcategui M, Garcia M, Sostre S, Garcia A. Subacute thyroiditis and dyserythropoesis after influenza vaccination suggesting immune dysregulation. Bol Asoc Med P R (2011) 103:48-52.

50. Hsiao J-Y, Hsin S-C, Hsieh M-C, Hsia P-J, Shin S-J. Subacute thyroiditis following influenza vaccine (Vaxigrip) in a young female. Kaohsiung J Med Sci (2006) 22:297-300. doi:10.1016/S1607-551X(09)70315-8

51. Girgis CM, Russo RR, Benson K. Subacute thyroiditis following the H1N1 vaccine. J Endocrinol Invest (2010) 33:506. doi:10.1007/BF03346633

52. Prebtani APH, Asa SL, Ezzat S. Is granulomatous thyroiditis a complication of breast implants? Endocr Pathol (2002) 13:239-44. doi:10.1385/EP:13:3:239

53. Vayssairat M, Mimoun M, Houot B, Abuaf N, Rouquette AM, Chaouat M. [Hashimoto's thyroiditis and silicone breast implants: 2 cases]. J Mal Vasc (1997) 22:198-9.

54. Naim JO, Lanzafame RJ, van Oss CJ. The effect of silicone-gel on the immune response. J Biomater Sci Polym Ed (1995) 7:123-32. doi:10.1163/1568562 95X00643

55. Willame C, Rosillon D, Zima J, Angelo M-G, Stuurman AL, Vroling H, et al. Risk of new onset autoimmune disease in 9- to 25-year-old women exposed to human papillomavirus-16/18 AS04-adjuvanted vaccine in the United Kingdom. Hum Vaccin Immunother (2016) 18:1-10. doi:10.1080/21645515. 2016.1199308

56. Costagliola S, Alcalde L, Tonacchera M, Ruf J, Vassart G, Ludgate M. Induction of thyrotropin receptor (TSH-R) autoantibodies and thyroiditis in mice immunised with the recombinant TSH-R. Biochem Biophys Res Commun (1994) 199:1027-34. doi:10.1006/bbrc.1994.1332

Conflict of Interest Statement: The authors declare that the research was conducted in the absence of any commercial or financial relationships that could be construed as a potential conflict of interest.

The reviewers IR and SF and handling Editor declared their shared affiliation, and the handling Editor states that the process nevertheless met the standards of a fair and objective review.

Copyright $\odot 2017$ Watad, David, Brown and Shoenfeld. This is an open-access article distributed under the terms of the Creative Commons Attribution License (CC BY). The use, distribution or reproduction in other forums is permitted, provided the original author(s) or licensor are credited and that the original publication in this journal is cited, in accordance with accepted academic practice. No use, distribution or reproduction is permitted which does not comply with these terms. 